PANORAMA

ISSN: $1909-7433$

ISSN: 2145-308X

ednorman@poligran.edu.co

Politécnico Grancolombiano

Colombia

\title{
USO DE RECURSOS EDUCATIVOS ABIERTOS EN MATEMÁTICAS PARA LA FORMACIÓN INTEGRAL DE ESTUDIANTES DE GRADO SÉPTIMO DE EDUCACIÓN BÁSICA SECUNDARIA
}

León Pereira, Carmen Cecilia; Heredia Escoza, Yolanda

USO DE RECURSOS EDUCATIVOS ABIERTOS EN MATEMÁTICAS PARA LA FORMACIÓN INTEGRAL DE ESTUDIANTES DE GRADO SEPTIMO DE EDUCACION BASICA SECUNDARIA

PANORAMA, vol. 14, núm. 26, 2020

Politécnico Grancolombiano, Colombia

Disponible en: http://www.redalyc.org/articulo.oa?id=343963784004

DOI: https://doi.org/10.15765/pnrm.v14i26.1481

Esta obra está bajo una Licencia Creative Commons Atribución-NoComercial-SinDerivar 4.0 Internacional 
Artículos de investigación científica y tecnológica

\section{USO DE RECURSOS EDUCATIVOS ABIERTOS EN MATEMÁTICAS PARA LA FORMACIÓN INTEGRAL DE ESTUDIANTES DE GRADO SÉPTIMO DE EDUCACIÓN BÁSICA SECUNDARIA}

PANORAMA, vol. 14, núm. 26, 2020

Politécnico Grancolombiano, Colombia

Recepción: 14 Junio 2019

Aprobación: 21 Febrero 2020

DOI: https://doi.org/10.15765/ pnrm.v14i26.1481

Redalyc: http://www.redalyc.org/ articulo.oa?id $=343963784004$

\author{
USE OF OPEN EDUCATIONAL RESOURCES IN \\ MATHEMATICS FOR THE INTEGRAL FORMATION \\ OF STUDENTS IN THE SEVENTH GRADE OF BASIC \\ SECONDARY EDUCATION \\ UTILIZAÇÃO DE RECURSOS EDUCATIVOS \\ ABERTOS EM MATEMÁTICA PARA A FORMAÇÃO \\ INTEGRAL DOS ALUNOS DO SÉTIMO ANO DO \\ ENSINO SECUNDÁRIO BÁSICO
}

\author{
Carmen Cecilia León Pereira A01307865@itesm.mx \\ Instituto Tecnológico de Monterrey, México \\ Yolanda Heredia Escoza yheredia@itesm.mx \\ Instituto Tecnológico de Monterrey, México
}

Resumen: Este artículo describe una investigación que tuvo como objetivo principal identificar el efecto del uso de Recursos Educativos Abiertos en matemáticas (REA), sobre la formación integral en estudiantes de séptimo grado de Educación Básica Secundaria, con edades entre 11 y 15 años. El estudio se desarrolló en una institución oficial, ubicada en Bucaramanga, departamento de Santander, Colombia. Siguiendo a Hernández Sampieri (citado por Hernández, Fernández y Baptista, 2010), se adoptó un enfoque mixto con un componente cuantitativo y otro cualitativo. En lo cuantitativo, el diseño fue expostfacto, no experimental, transeccional descriptivo, se aplicó una escala Likert que averiguó sobre las competencias de aprendizaje permanente, entre las cuales se incluyeron competencias matemáticas, manejo de información, manejo de situaciones de la vida y convivencia. Se aplicó también un cuestionario para ahondar sobre aprendizaje colaborativo. El componente cualitativo permitió un acercamiento para indagar sobre tres aspectos del fenómeno de estudio: aprendizaje mediado por tecnología, aprendizaje colaborativo y aprendizaje de matemáticas. Los hallazgos muestran evidencias de que la incorporación pedagógica de REA en el currículo permite el fortalecimiento de las competencias integrales. Estas evidencias pueden ser analizadas tanto por la comunidad educativa de la institución -en donde ese realizó la investigación-, como por otras instituciones afines que también ofrecen educación básica y media e incorporan REA en matemáticas, con el fin de enriquecer sus proyectos curriculares y de capacitación de docentes sobre los beneficios y posibilidades del uso pedagógico de REA. Palabras clave: Competencia, competencias integrales, aprendizaje colaborativo, competencias matemáticas, recursos educativos abiertos.

Abstract: This article describes a research that had as main objective: to identify the effect of the use of Open Educational Resources in Mathematics (OER), on the integral formation of students of seventh grade of Basic Secondary Education, with 
ages between 11 and 15 years old. The study was carried out in an official institution, located in Bucaramanga, Santander Department, Colombia. Following Hernández Sampieri (quoted by Hernández, Fernández and Baptista, 2010) a mixed approach was adopted with a quantitative and a qualitative component. Quantitatively, the design was expostfacto, non-experimental, transectional and descriptive. A Likert scale was applied to find out about the competencies: lifelong learning, among which were included Mathematical competencies, information management, management of life situations and coexistence. A questionnaire was also applied to further explore collaborative learning. The qualitative component allowed an approach to investigate three aspects of the study phenomenon: technology-driven learning, collaborative learning and mathematical learning. The findings show evidence that the pedagogical incorporation of AER in the curriculum allows for the strengthening of comprehensive competencies. This evidence can be analyzed both by the educational community of the institution where the research was carried out and by other related institutions that also offer basic and secondary education and incorporate AER in Mathematics, in order to enrich their curricular and teacher training projects on the benefits and possibilities of the pedagogical use of AER.

Keywords: Competence, integral competences, collaborative, learning, mathematical, competences, open educational resources.

Resumo: Este artigo descreve uma investigação que teve como principal objectivo: identificar o efeito da utilização de Recursos Educativos Abertos em Matemática (REA), na formação integral dos alunos do $7^{\circ}$ ano do Ensino Básico Secundário, com idades compreendidas entre os 11 e os 15 anos. $\mathrm{O}$ estudo foi realizado numa instituição oficial, localizada em Bucaramanga, Departamento de Santander, Colômbia. $\mathrm{Na}$ sequência de Hernández Sampieri (citado por Hernández, Fernández e Baptista, 2010) foi adoptada uma abordagem mista com uma componente quantitativa e uma componente qualitativa. Quantitativamente, o desenho foi expositivo, não experimental, transeccional e descritivo, tendo sido aplicada uma escala de Likert para conhecer as competências: aprendizagem ao longo da vida, entre as quais se incluem competências matemáticas, gestão da informação, gestão de situações da vida e coexistência. Foi também aplicado um questionário para aprofundar a aprendizagem em colaboração. A componente qualitativa permitiu uma abordagem para investigar três aspectos do fenómeno do estudo: aprendizagem orientada para a tecnologia, aprendizagem em colaboração e aprendizagem matemática. Os resultados mostram que a incorporação pedagógica da AER no currículo permite o reforço de competências abrangentes. Esta evidência pode ser analisada tanto pela comunidade educativa da instituição onde a investigação foi realizada como por outras instituições relacionadas que também oferecem ensino básico e secundário e incorporam a AER em Matemática, de forma a enriquecer os seus projectos curriculares e de formação de professores sobre os benefícios e possibilidades do uso pedagógico da AER.

Palavras-chave: Competência, competências integrais, aprendizagem em colaboração, competências matemáticas, recursos educativos abertos.

\section{INTRODUCCIÓN}

La denominada sociedad del conocimiento en que vivimos se desenvuelve en contextos complejos que demandan el cultivo pleno de potencialidades del ser humano para asumir retos importantes en la toma de decisiones respecto a cosas fundamentales como elegir una profesión sin conocer los empleos futuros, crear una empresa ética y sostenible, organizar una familia, estudiar y trabajar en ambientes multiculturales y hacer un uso acertado del tiempo. Retos semejantes han existido, pero actualmente se hacen mayores por las incertidumbres de que habla Morin (1999), en 
su documento Los siete saberes necesarios para la educación del futuro: incertidumbres de lo real, del conocimiento y de la ecología de la acción.

Esos contextos complejos tienen entre sus características desarrollos como las tecnologías de la información y la comunicación (TIC), que ofrecen diversas posibilidades de innovación en muchos campos de la vida social y educativa. En este segundo campo, las TIC pueden enriquecer los ambientes de aprendizaje, pero se requiere que se haga un uso planeado, intencional y con revisión y análisis de sus efectos sobre los procesos de formación de los estudiantes. Porque la innovación tecnológica no conduce, por sí sola, a mejoras pedagógicas o efectos formativos deseados que, según Díaz-Barriga (2011), llevan a transformar lo que se sabe y no solo a repetirlo. Identificar esos efectos fue el objetivo principal de la investigación presentada en este artículo.

Teniendo en cuenta esa realidad tecnológica, en la educación básica y media se han venido impulsando propuestas para ofrecer una educación integral, enfocada en el desarrollo de competencias integrales (CI). El Ministerio de Educación Nacional (MEN), ha publicado diversos documentos y promulgado disposiciones orientados a motivar al sector educativo hacia la adopción de la tecnología como un elemento apropiado para el aprendizaje de las áreas fundamentales de la educación básica y media (MEN, 2008). Una de esas áreas es la de Matemáticas, que fue la elegida para realizar la parte metodológica de esta investigación. Si se tiene en cuenta la realidad educativa, es necesario reconocer que existe lentitud en las escuelas para adoptar la tecnología. Según Gates (1995), dicha lentitud puede ser debida a una tendencia bastante generalizada al conservadurismo o a falta de recursos.

También circulan en el sector educativo diversos documentos que señalan la importancia de emplear las TIC en los procesos educativos y que dan cuenta de beneficios que pueden obtenerse con ello (López y Hederich, 2010). Al mismo tiempo, hace falta verificar la consecución de resultados (López, 2019). Identificar y analizar evidencias de las vivencias de los estudiantes mientras participan en actividades de formación integral con ayuda de recursos tecnológicos fue un aspecto central de esta investigación.

Las búsquedas de esas evidencias se realizaron con base en un enfoque de desarrollo de CI, sin desconocer que el enfoque de competencias ha sido muy controvertido (Díaz-Barriga, 2011). Esta controversia ha sido tenida en cuenta proactivamente para dotarlo de un carácter educativo y humanizante. Al respecto, Tobón (2004) aporta el concepto de la formación basada en competencias como una propuesta educativa orientada a la formación integral humana, que permite integrar la teoría con la práctica, mejorar la convivencia, orientar proyectos de vida y articular los niveles del sistema educativo. Este autor hace un aporte importante en el esfuerzo de humanizar el enfoque en mención, porque propone considerar tres dimensiones de las competencias, así: afectivo-motivacional relacionada con actitudes y valores; cognoscitiva sobre conocimientos factuales, conceptos y habilidades cognitivas y la actuacional sobre habilidades procedimentales y técnicas. Perrenoud 
(2009) analiza potencialidades y limitaciones de la formación por competencias y señala la importancia del manejo interdisciplinar del conocimiento.

El proyecto educativo de la institución en la cual se realizó la investigación tiene un enfoque pedagógico de formación que es coherente con la búsqueda del desarrollo de CI en todas las áreas curriculares. A nivel local, en matemáticas existe disponibilidad de Recursos Educativos Abiertos (REA), y también se puede acceder a repositorios organizados en otros países, aprovechando las ventajas del movimiento educativo abierto. Estas son condiciones para interesarse por su aplicación pedagógica y por los efectos de su empleo en el aprendizaje y como ayudas para resolver dificultades que frecuentemente se presentan en Matemáticas. Este tema ha sido estudiado por Aragón, Castro, Gómez y González (2009). Y los informes de la Organización para la Cooperación y el Desarrollo Económico (OCDE), correspondientes a los años 2012 y 2016 sobre la prueba PISA revelan que Colombia, aunque alcanzó una leve mejoría en 2015, sigue siendo uno de los países con promedios bajos en matemáticas; también la institución educativa en donde se desarrolla la investigación requiere buscar estrategias en busca de mejores resultados.

La existencia de recursos tecnológicos aprovechables en educación, el propósito de formación integral de la institución seleccionada, las falencias en el aprendizaje de las matemáticas y la necesidad de evaluar los efectos del empleo de TIC por medio de Recursos Educativos Abiertos (REA), son aspectos que justificaron la investigación. De acuerdo con los alcances de la misma, se eligió el área de Matemáticas, en la cual una docente había iniciado una experiencia de empleo de dichos materiales.

Entre muchos estudios que anteceden esta investigación es posible señalar varios, como los que se mencionan a continuación. Ambientes de aprendizaje (Jaramillo y Ruiz, 2010). Procesos de implementación de REA y de objetos de aprendizaje (Mortera, 2011). Desarrollar en los individuos la capacidad de realizar cosas nuevas, ser creativos, descubridores, y aprender permanentemente, como objetivo de la educación del siglo XXI (Ramírez, Huerta y Flórez, 2012). Incremento de la motivación de los estudiantes debida a la realización de actividades auténticas en un contexto cercano al real (Naeemi, Zare, Hormozi, Shaqaqi, y Kaveh, 2011). Uso de REA y objetos de aprendizaje para mejorar las competencias matemáticas (Pazos, Tenorio y Ramírez, 2015). Competencias para la resolución de problemas en matemáticas (Avilés, Díaz, Esquivel y Hernández, 2010). Aprendizaje colaborativo basado en TIC (García-Varcárcel, Hernández, Muñoz-Repiso, 2012).

\section{MARCO TEÓRICO}

En los sectores educativos de las cambiantes y complejas sociedades actuales se viene tomando conciencia de la necesidad de formar personas que conozcan, valoren y cultiven sus capacidades; sean creativas, estén dispuestas a aprender permanentemente y posean las habilidades y criterios necesarios para aprovechar los recursos tecnológicos en el 
tratamiento y resolución de asuntos y problemas relacionados con el trabajo, la vida misma y el bien común. Satisfacer expectativas como esas tiene como condición necesaria, aunque no suficiente, que los sistemas educativos promuevan una formación de los educandos acorde con las exigencias y las posibilidades actuales.

Como respuesta a esa realidad, existen múltiples ofertas para el pleno desarrollo de las potencialidades humanas, entre las cuales están las de educación integral, que se han venido posicionando en la cultura escolar impulsadas por los avances logrados por Gardner (1988) y sus colaboradores, en la ciencia cognitiva, especialmente en psicología. A las anteriores se suman las ofertas de comunicación permanente y global mediante las TIC, que tienen incidencia en la conformación de la identidad social de niños, jóvenes y adultos. Además, los medios de comunicación y las redes sociales ofrecen facilidades de acceso a los más diversos conocimientos. En ese contexto se procura hoy una formación enfocada al desarrollo de CI.

\section{Competencias y enfoque de formación por competencias}

En la educación formal, el empleo del término competencia se inició en el área de lenguaje en el año 1960, y a partir de entonces se han elaborado construcciones teóricas y modelos para su aplicación; estos se han difundido masivamente en las políticas educativas de numerosos países. Según López (2014), el término competencia tiene dos matices o significados, cuya etimología es latina: uno que proviene del verbo cumpetere como competer; es decir, saber y saber hacer de manera experta, y el otro proviene de com-petitum como competir, saber ser, superación, esfuerzo. Así, la competencia es suma de saber, saber hacer y saber ser.

Entre los educadores existen posiciones antagónicas sobre la incorporación del enfoque de competencias en educación. Díaz-Barriga (2011) refiere que algunos lo consideran opuesto al ideal de formación integral de un individuo y con gran orientación al mundo del trabajo y a la competitividad. Tobón (2004), plantea que dichas posiciones han generado discusiones durante los últimos decenios y han dificultado la adopción del enfoque mencionado en los currículos de educación formal.

\section{Competencias integrales y formación integral}

El tema de competencias integrales es amplio y su conceptualización puede hacerse desde variados enfoques con diferentes nombres, dada la naturaleza de las instituciones que aportan dicha conceptualización. Sin embargo, al analizar los contenidos que tratan es posible encontrar coincidencia en la orientación hacia la formación integral de los estudiantes. Ejemplo de esa coincidencia lo constituyen las propuestas de Delors, Tobón, del MEN y de la OCDE.

Para Tobón (2004), la formación basada en competencias es una propuesta educativa orientada a la formación integral humana; permite 
fusionar la teoría con la práctica y mejorar la convivencia; facilita la articulación entre los niveles del sistema educativo y orienta un proyecto de vida.

Delors (1996), en su propuesta, que es ampliamente conocida en los contextos académicos, organiza en cuatro pilares las misiones que tiene actualmente la educación: aprender a conocer, aprender a hacer, aprender a vivir juntos y aprender a ser. Sobre esos cuatro pilares es posible construir la formación integral de la niñez y la juventud.

Para este autor, el aprender a conocer implica aprender a aprender, ejercitar la memoria y el pensamiento y adquirir las bases para seguir aprendiendo. El aprender a hacer está unido al aprender a conocer e implica capacidad para integrar teoría y práctica, para establecer relaciones estables y eficaces entre las personas y para manejar la incertidumbre.

El aprender a vivir juntos se manifiesta en la capacidad de participar en proyectos comunes, así como en la aplicación de métodos para evitar o resolver conflictos. Y el cuarto pilar, el aprender a ser, se refiere al conocimiento de sí mismo y de los otros y a la apertura hacia ellos; esto conlleva el despliegue completo de los talentos, actuaciones autónomas, cultivo de memoria, razonamiento, sentido estético, capacidades físicas y aptitudes para comunicar.

En síntesis, es posible afirmar que para Delors las competencias integrales entretejen conocimientos, capacidades, actitudes, valores y habilidades como cualidades que se han de desarrollar en una persona cuando se la considera y se forma como un todo. Los cuatro tipos de aprendizajes constituyen lo que se ha llamado competencia integrada.

De los planteamientos de la OCDE (2005) es posible inferir que para esta organización la persona competente actúa eficazmente porque integra y moviliza habilidades prácticas, conocimientos, motivaciones, valores, sentimientos y actitudes. Señala, además, que un amplio rango de esas competencias resulta clave para que el ser humano pueda enfrentar idóneamente los desafíos actuales. Las organiza en los siguientes grupos: uso interactivo de herramientas, interacción entre grupos heterogéneos y actuar autónomamente. Enuncia tres dimensiones que se deben tener en cuenta en el desarrollo de esas competencias: información, comunicación e impacto ético-social. A cada una de esas dimensiones le asigna unos contenidos. En la dimensión de información incluye el acceso, la evaluación y la organización de la información y la investigación y la resolución de problemas. En la dimensión de comunicación destaca la capacidad de comunicar, intercambiar, criticar, presentar información y el uso de aplicaciones de TIC. Y la dimensión de impacto ético-social la desglosa en actuar con efecto social positivo, aplicar criterios para usar responsablemente las TIC, poseer pensamiento crítico y tener capacidad de tomar decisiones.

En Colombia, tanto el Congreso de la República como el MEN han producido normas y documentos en los cuales establecen disposiciones y orientaciones para el desarrollo de las competencias a las cuales les dan varios nombres. El Congreso aprobó, en 1994, la Ley 115 que es conocida como Ley General de Educación. El artículo 5 de la misma 
establece los fines de la educación colombiana, y el artículo 92 esboza lo que puede considerarse un perfil de ciudadano de este país. Ambos artículos proponen competencias que, en términos generales, coinciden con las planteadas por Delors, la OCDE y otros autores antes señalados.

Para llevar a cabo la implementación de la Ley 115, el MEN ha diseñado y entregado al país numerosos documentos, entre los cuales están: Estándares Básicos de Competencias en Lenguaje, Matemáticas, Ciencias y Ciudadanía (Documento 3, 2006). La Guía No. 21: articulación de la educación con el mundo productivo (2005), y la Guía 30: ser competente en tecnología (2009).

En los tres documentos, el concepto de competencias es coincidente y concordante con lo presentado anteriormente respecto de lo propuesto por Delors y la OCDE. En el informe titulado Revolución Educativa 2002-2010, Acciones y Lecciones, el MEN (2010) agrupa bajo el nombre de CI las siguientes: básicas (científicas, ciudadanas, comunicativas y matemáticas), laborales y profesionales. Esta postura se observa también en MEN (2005).

El MEN (2008) señala algunos desafíos que deben afrontarse en la formación en tecnología: incrementar la motivación para estimular la curiosidad científica y tecnológica; reconocer que el saber tecnológico contribuye a resolver problemas y a transformar el entorno; reflexionar críticamente sobre las relaciones entre tecnología y sociedad; facilitar la vivencia de actividades relacionadas con el conocimiento tecnológico y proveer a las personas de herramientas para participar activamente en su entorno.

Por todos los planteamientos antes expuestos se consideran las competencias integrales un conjunto de conocimientos, habilidades, actitudes, valores que adquiere y cultiva un individuo, mediante los cuales atiende las demandas complejas del diario vivir.

\section{Aprendizaje colaborativo}

La interacción humana es cada vez más valorada en contextos familiares, escolares, empresariales, deportivos y comerciales. En particular, lo es intencionalmente en establecimientos educativos cuando se la emplea como una estrategia de integración humana que puede mejorar los procesos de aprendizaje, contribuir al conocimiento mutuo, facilitar la resolución de problemas, posibilitar la realización de proyectos de interés común y fomentar el aprendizaje permanente. El diálogo entre personas y entre grupos resulta útil para lograr cambios a nivel colectivo en la manera de pensar y para unir voluntades con el propósito de conseguir metas concertadas.

Las teorías del aprendizaje, al tratar la importancia de la interacción en los procesos de enseñanza, señalan que cuando hay colaboración los integrantes de un grupo realizan intercambios de esfuerzos, de modo que el alcanzar un objetivo común genere beneficios para cada uno (GarcíaValcárcel, Hernández y Muñoz-Repiso. 2012). 
Con base en sus investigaciones, Fernández-Cárdenas (2011) reconoce que la calidad de la educación depende de los procesos que se dan en el aula y propone la perspectiva socio histórica para definir con detalle aspectos relevantes, haciendo énfasis en elementos como socialización, mediación, negociación de significado y comunidad. Todos estos aspectos son relevantes dentro del aprendizaje colaborativo.

De acuerdo con Daza-Orozco (2015) puede decirse que el aprendizaje colaborativo es una estrategia de interacción humana. Mediante el diálogo, los grupos de personas pueden lograr cambios a nivel colectivo en la manera de pensar y unir esfuerzos para alcanzar metas comunes con el objeto de maximizar el aprendizaje.

\section{Competencias matemáticas}

Los aportes que pueden obtenerse de la educación matemática a la formación integral y, por consiguiente, al desarrollo de CI son un tema de debate en círculos académicos, sociales y familiares. El MEN, en el Documento $\mathrm{N}^{\circ} 3$ (2006) participa en las discusiones y afirma:

La educación matemática debe responder a nuevas demandas globales y nacionales, como las relacionadas con una educación para todos, la atención a la diversidad y a la interculturalidad y la formación de ciudadanos y ciudadanas con las competencias necesarias para el ejercicio de sus derechos y deberes democráticos. (p. 46)

En el mismo documento, el MEN sustenta su afirmación con argumentos relacionados con el papel que han jugado las Matemáticas en el arte, las artes plásticas, la ingeniería, la economía y el comercio. En otro argumento señala las contribuciones de esa disciplina a la formación del pensamiento lógico y al desarrollo de la ciencia y la tecnología.

El citado documento del MEN profundiza el análisis sobre algunos aspectos de la educación matemática; sustenta la idea de que los procesos de aprendizaje de las matemáticas no son algo aislado, sino que implican componentes de orden social y afectivo, ligados con entornos de aprendizaje específicos, relacionando así las matemáticas con los fines culturales de la educación. Resalta la importancia de trabajar en la clase en procura de crear una comunidad de aprendizaje mediante el interactuar de docentes y estudiantes para favorecer el desarrollo del pensamiento crítico, la iniciativa y la validación del conocimiento.

Las competencias matemáticas han sido conceptualizadas también por la OCDE. En el informe de la prueba PISA del año 2006 considera que son una clase de capacidades que adquieren las personas y que les permiten razonar, comprender, investigar, argumentar y comunicar respecto al papel que juegan las matemáticas en asuntos de la vida individual y colectiva y que les facilitan la aplicación del razonamiento matemático en la solución de problemas (2013).

En este trabajo se indagó por las competencias de recuperación de información, razonamiento, reconocimiento de reglas simples, resolución de problemas. 
Los REA son recursos digitales disponibles por medio de Internet, de manera gratuita, para docentes, estudiantes e investigadores. Entre ellos se encuentran documentos de texto, audio, video, juegos educativos, portales, cursos completos, materiales de cursos y herramientas de software que facilitan el acceso al conocimiento. Se desarrollan a través de las TIC y por este medio se posibilita su uso con fines no comerciales (Mortera, 2011). Los REA se caracterizan por ser fáciles de localizar, reusables, se pueden adaptar a distintos ordenadores, obtener un correcto funcionamiento y beneficiarse de la existencia de repositorios. Existe el Movimiento Educativo Abierto, el cual busca que el conocimiento esté al alcance de un mayor número de personas para disminuir la brecha entre países, con recursos de calidad (Mortera, 2011).

La investigación se centró en el desarrollo de CI con actividades propias del área seleccionada apoyadas con los siguientes REA: Geogebra, Probabilidad, Teorema de Pitágoras, Graficadores de Estadística Descriptiva y el empleo de la plataforma social Edmodo.

Con base en los planteamientos sobre la importancia del desarrollo integral de niños y jóvenes, de las posibilidades que ofrecen los recursos tecnológicos a la educación y de la conveniencia de emplearlos en el área de Matemáticas para mejorar el aprendizaje, surgió la siguiente pregunta de investigación: ¿Cuál es el efecto del uso de REA en matemáticas, mediados por TIC, sobre la formación integral de estudiantes de séptimo grado de educación básica secundaria (EBS)?

Esta pregunta se especificó con las siguientes preguntas subordinadas: ¿Qué nivel de aceptación reportan los estudiantes de séptimo grado de EBS, en relación con el uso de TIC sobre el desarrollo de las CI? ¿Cuáles beneficios reportan las estudiantes de séptimo grado de EBS, como obtenidos mediante el uso de REA en actividades de aprendizaje colaborativo en matemáticas? ¿Cuáles son los efectos en el aprendizaje que reconocen los estudiantes de EBS acerca del uso de REA en matemáticas?

Las anteriores preguntas se plantearon buscando cumplir los siguientes objetivos:

Objetivo general. Identificar el efecto del uso de REA en matemáticas, mediado por TIC sobre la formación integral de estudiantes de séptimo grado de EBS.

Objetivos específicos. Explicitar el nivel de aceptación que manifiestan los estudiantes de séptimo grado de EBS, acerca del empleo de TIC en ambientes mediados por tecnologías y orientado a la formación integral en aprendizaje permanente, manejo de información y de situaciones de la vida y competencias para la vida y la sociedad.

Identificar beneficios que reportan los estudiantes de séptimo grado de EBS como obtenidos mediante el uso de los REA en actividades de aprendizaje colaborativo utilizando TIC en desarrollo de habilidades comunicativas orales, interacción con el otro, valoración de las tutorías, ayuda mutua. 
Inferir características de REA en el aprendizaje de matemáticas mediado por TIC y orientado a recuperación de información, comprensión y aprendizaje de conceptos, empleo de algoritmos y motivación por el aprovechamiento pedagógico de la tecnología, que reconocen los estudiantes de séptimo grado de EBS, como vivenciadas con su uso interactivo.

Para encontrar respuestas a los cuestionamientos se realizó una investigación, para la cual se adoptó un enfoque mixto con un componente cuantitativo y otro cualitativo.

\section{MÉTODO E INSTRUMENTOS}

Entre los enfoques que podían ser adoptados para realizar esta investigación se escogió el mixto que Hernández Sampieri (citado por Hernández, Fernández y Baptista, 2010), caracteriza como aquel que busca aprovechar las fortalezas tanto de la indagación cuantitativa como las de la cualitativa; las combina y procura reducir sus debilidades. Trata en un mismo estudio, por lo menos, un componente cuantitativo y otro cualitativo; logra los objetivos mediante procesos sistemáticos, rigurosos y críticos de recolección de datos para cada componente y de análisis e integración de resultados, de modo que sea posible realizar inferencias que ayuden a obtener una mayor comprensión del hecho, fenómeno o situación estudiada.

\section{Diseño del componente cuantitativo}

Para el componente cuantitativo de la investigación se eligió el diseño transeccional, no experimental, descriptivo. Por ser no experimental, la investigación se realizó sin una manipulación deliberada de variables, ya que fundamentalmente se propuso observar y describir hechos o fenómenos tal como se dan en un contexto determinado y recolectar información sobre las variables para luego analizarla (Dzul-Escamilla, 2013). Por ser transeccional, los datos cuantitativos se recolectaron en un único momento, pues se buscó describir las variables de interés y analizar, en un momento dado, el efecto del empleo de REA en el desarrollo de las competencias objeto de estudio. En este componente se aplicaron una escala Likert y un cuestionario.

\section{Diseño del componente cualitativo}

El diseño del componente cualitativo estuvo constituido por la preparación de técnicas que permitieron recolectar la información en contacto con los estudiantes participantes mediante entrevistas semiestructuradas, un cuestionario y una guía para la observación en clase con participación moderada. Se buscó interpretar el significado de las experiencias de los estudiantes con respecto al uso de REA en matemáticas 
para complementar la información sobre la incidencia de su empleo en el desarrollo de CI.

En este componente las categorías fueron aprendizaje mediado por REA con los indicadores utilidad, desempeño autorregulado y motivación. Competencias matemáticas con recuperación de información, reconocimiento de reglas simples, razonamiento lógico; y aprendizaje colaborativo con los indicadores interacción, retroalimentación, bien común, compañerismo.

Priorización de uno de los componentes

Se priorizó el componente cuantitativo, que permitió obtener un panorama general del fenómeno investigado y se profundizó en algunas competencias objeto del estudio, mediante el enfoque cualitativo. En su conjunto se trató de un diseño concurrente (Pereira, 2011).

\section{Procedimiento}

La investigación se enfocó en los principios éticos que presentan Valenzuela y Flores (2012), respecto a propósito, duración, procedimientos a seguir, personas a contactar y consentimientos informados. En las clases de matemáticas de séptimo grado se continuó el desarrollo de las sesiones de Geogebra y los docentes realizaron otras prácticas contextualizadas, con todos los participantes, en las cuales ellos interactuaron con los REA seleccionados. Se aplicaron los cuestionarios sobre Competencias Integrales y Tecnológicas y la encuesta sobre Aprendizaje Colaborativo a la muestra de 76 estudiantes. Se realizaron tres observaciones a grupos de clase. Al terminar dichas prácticas se llevaron a cabo otras sesiones con los siete estudiantes de la muestra intencional, a quienes se les realizaron las entrevistas semiestructuradas.

Los datos cuantitativos obtenidos con la escala Likert y la encuesta se capturaron y procesaron con el programa SPSS. Las respuestas a las preguntas abiertas de la encuesta sobre aprendizaje colaborativo se capturaron en tablas de Excel con el propósito de lograr una reducción de datos atendiendo criterios de afinidad. Se transcribieron las entrevistas y se utilizó la guía de observación para el análisis respectivo. Sobre la validez interna y externa se cuidaron las causas de invalidación que señalan Valenzuela y Flores (2012).

El componente cualitativo incluyó 21 entrevistas con siete estudiantes, tres entrevistas a cada uno. Cada entrevista tuvo como tema uno de los siguientes REA: Introducción al concepto de probabilidad; Ejercicios prácticos interactivos de probabilidad, Teorema de Pitágoras, explicaciones básicas ilustrativas; Teorema de Pitágoras, ejercicios ilustrativos; Teorema de Pitágoras, ejercicios prácticos interactivos y Geogebra.

La confiabilidad del estudio se buscó a partir de que los instrumentos empleados midieran o indagaran con certeza por el objeto de estudio; se utilizaron instrumentos con permisos de uso, previamente validados por los autores. Se realizaron algunas adaptaciones según el contexto. 


\section{Instrumentos empleados}

Para la recolección de la información se emplearon cuatro instrumentos, todos debidamente validados en estudios previos. Los autores concedieron las correspondientes autorizaciones para que fueran utilizados en esta investigación. Tres de los cuatro instrumentos requirieron adaptaciones menores en relación con la disciplina en la cual se desarrolló este estudio y la edad de los estudiantes. Los instrumentos fueron:

Competencias Integrales y Tecnológicas. Es un instrumento de Suárez, Rodríguez y Rodríguez (2012). Está estructurado en la forma siguiente: la primera parte indaga sobre el uso de la tecnología y, en segundo lugar, un cuestionario que tiene cuatro apartados sobre CI y fue elaborado con la técnica de Likert. Esos apartados se refieren a competencias para logro de aprendizaje permanente, manejo de la información, manejo de situaciones de la vida y competencias para la convivencia y vida en sociedad. Dicho instrumento se aplicó para recolectar información de la opinión de estudiantes sobre CI puestas en acción en un ambiente de aprendizaje mediado por tecnología.

Encuesta sobre el aprendizaje colaborativo. Es un cuestionario que combina preguntas cerradas con preguntas abiertas; fue construido por Martínez, Catalá y Díaz (2013).

Entrevista Semiestructurada REA. Creada por Asprilla (2012), es una guía para una entrevista de preguntas abiertas.

Guia de Observación de clase. La observación se realizó en la modalidad participación moderada, descrita por Valenzuela y Flores (2012). Como guía se utilizó el modelo de Alvarado (2012).

En el componente cuantitativo no experimental, transeccional descriptivo, se aplicaron dos cuestionarios siguiendo a Valenzuela y Flores (2012), quienes expresan que, en el diseño transeccional descriptivo, se realizan observaciones en un solo momento en el tiempo y se cuantifican las variables individualmente sin describir relaciones entre ellas.

\section{POBLACIÓN Y MUESTRA}

La población de interés para este estudio estuvo conformada por 96 estudiantes de grado séptimo, género femenino, de una institución educativa oficial, quienes han trabajado previamente en sus clases con el software libre Geogebra, en una asignatura que integra matemáticas con el uso de las TIC. Las estudiantes continuaron utilizando este software en geometría y estadística y otros REA que los docentes incorporaron a las prácticas de clase y se inició el uso de la plataforma Edmodo.

Siguiendo a Hernández, Fernández y Baptista (2010), se seleccionó una muestra probabilística de 76 estudiantes para el componente cuantitativo, tamaño obtenido con el software Stats 2.0, con un 5\% de error y un $95 \%$ de confiabilidad. La selección de las estudiantes se llevó a cabo dentro de las aulas de clase, utilizando una tómbola sin reemplazo, en la cual todas las estudiantes tuvieron la misma probabilidad de ser seleccionadas. Para las 
entrevistas, la muestra fue intencional de siete participantes; se incluyeron estudiantes de distintos niveles de desempeño.

\section{RESULTADOS}

Como el enfoque de la investigación fue mixto, se presentan los resultados obtenidos tanto para el componente cuantitativo como para el cualitativo. La primera parte de los resultados muestra una síntesis de la información obtenida al procesar las respuestas que las participantes dieron al instrumento de Suárez y otros (2012) sobre el Uso de la tecnología. Los demás hallazgos se presentan organizados de acuerdo con los objetivos del estudio y se concluye con la respuesta que se encontró para la pregunta formulada inicialmente.

\section{Uso de la tecnología}

Se consultó, a las 76 estudiantes de la muestra acerca de la disponibilidad y el uso de tecnologías, que es un aspecto decisivo para el desarrollo de competencias tecnológicas. Los hallazgos muestran que el 95\% de las participantes tiene computadora en casa; algunas tienen un solo equipo y otras tienen dos. El 96\% tiene conexión a Internet desde su casa y más de la mitad tiene modalidad inalámbrica. Solo el $4 \%$ no tiene Internet en casa, pero recurre a las conexiones que tienen amigos y familiares. El tiempo de uso diario de Internet para estudiar las materias curriculares varía entre menos de una hora $(18,4 \%)$, y más de dos $(17,1 \%)$. Parece que, si bien entre las estudiantes que diligenciaron el instrumento hay acceso generalizado a esa ayuda tecnológica, para algunas hacen falta todavía mayores períodos de aprovechamiento de la misma, si se tiene en cuenta que la mayoría tiene computador en su residencia.

\section{Resultados para el primer objetivo correspondiente al componente cuantitativo}

El objetivo fue: Explicitar el nivel de aceptación que manifiestan las estudiantes de séptimo grado de EBS, acerca del uso de REA matemáticas en ambientes mediados por tecnologías y orientado a la formación integral en aprendizaje permanente, manejo de información, de situaciones de la vida y competencias para la convivencia y vida en sociedad.

La indagación sobre esas cuatro CI se llevó a cabo mediante la aplicación del instrumento, ya mencionado, de Suárez y otros (2012). En ellos se desglosa cada CI en un grupo de competencias para su valoración según escala Likert. Las opciones de respuesta y su valoración numérica son: Muy de acuerdo 5. De acuerdo 4. Ni de acuerdo ni en desacuerdo 3. En desacuerdo 2, y Muy en desacuerdo 1. En las Tablas 1 y 2 se aprecia el resultado general. 


\begin{tabular}{|c|c|c|c|c|c|c|c|c|c|c|}
\hline \multirow{2}{*}{$\begin{array}{l}\text { Competen } \\
\text { cia }\end{array}$} & \multirow{2}{*}{ Competencia } & \multirow{2}{*}{$N$} & \multirow{2}{*}{$\begin{array}{c}\text { Ran } \\
\text { go }\end{array}$} & \multirow{2}{*}{$\begin{array}{c}\text { Mini } \\
\text { mo }\end{array}$} & \multirow{2}{*}{$\begin{array}{l}\text { Máx } \\
\text { mo }\end{array}$} & \multirow{2}{*}{ Media } & \multirow{2}{*}{$\begin{array}{c}\text { Desv. } \\
\text { Desviación }\end{array}$} & \multicolumn{2}{|c|}{ Asimetria } & \multirow{2}{*}{$\begin{array}{l}\text { Orden } \\
\text { según } \\
\text { la } \\
\text { Media }\end{array}$} \\
\hline & & & & & & & & Estadistico & $\begin{array}{l}\text { Desv. } \\
\text { Error }\end{array}$ & \\
\hline \multirow{5}{*}{ 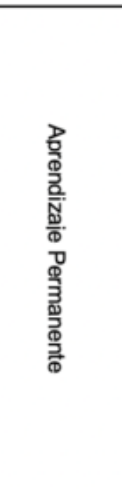 } & $\begin{array}{l}\text { 4.Resolución de } \\
\text { Problemas }\end{array}$ & 76 & 4 & 1 & 5 & 3,96 & 0,871 & $-0,793$ & 0,276 & 12 \\
\hline & $\begin{array}{l}\text { 5.Razonar } \\
\text { lógicamentente y } \\
\text { comunicar } \\
\text { 6.Inciarse en }\end{array}$ & 76 & 4 & 1 & 5 & 3,96 & 0,916 & $-0,991$ & 0,276 & 13 \\
\hline & $\begin{array}{l}\text { tecnología y } \\
\text { continuar } \\
\text { autónomamente } \\
\text { 7.Admitir } \\
\text { diversidad de }\end{array}$ & 76 & 4 & 1 & 5 & 4,16 & 0,88 & $-0,92$ & 0,276 & 8 \\
\hline & $\begin{array}{l}\text { respuestas y } \\
\text { motivarse para } \\
\text { buscar distintos } \\
\text { enfoques }\end{array}$ & 76 & 4 & 1 & 5 & 3,87 & 0,854 & $-0,929$ & 0,276 & 16 \\
\hline & $\begin{array}{l}\text { 8.Aprender, } \\
\text { asumir y dirigir su } \\
\text { aprendizaje }\end{array}$ & 76 & 4 & 1 & 5 & 4,24 & 0,892 & $-1,068$ & 0,276 & 3 \\
\hline \multirow{4}{*}{ 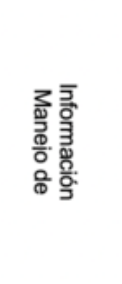 } & $\begin{array}{l}\text { 9.Búsqueda e } \\
\text { identificación }\end{array}$ & 76 & 4 & 1 & 5 & 4,28 & 0,842 & $-1,254$ & 0,276 & 2 \\
\hline & $\begin{array}{l}\text { 10.Evaluación, } \\
\text { selección y } \\
\text { sistematización }\end{array}$ & 76 & 4 & 1 & 5 & 3,93 & 0,984 & $-0,985$ & 0,276 & 14 \\
\hline & $\begin{array}{l}\text { 11.Analizar, } \\
\text { sintetizar, utilizar, } \\
\text { compartir }\end{array}$ & 76 & 4 & 1 & 5 & 4,3 & 0,766 & $-1,311$ & 0,276 & 1 \\
\hline & $\begin{array}{l}\text { 12.Procesar y } \\
\text { comunicar }\end{array}$ & 76 & 4 & 1 & 5 & 4,12 & 0,993 & $-1,167$ & 0,276 & 9 \\
\hline
\end{tabular}

\section{Tabla 1}

Resultado de escala Likert Competencias Integrales - Aprendizaje permanente y Manejo de información Fuente: elaboración propia. 
Tabla 2
Resultado de escala Likert Competencias Integrales - Manejo de Situaciones y Convivencia

Fuente: elaboración propia.

\begin{tabular}{|c|c|c|c|c|c|c|c|c|c|c|}
\hline \multirow{2}{*}{$\begin{array}{c}\text { Competen } \\
\text { cia }\end{array}$} & \multirow{2}{*}{ Competencia } & \multirow{2}{*}{$N$} & \multirow{2}{*}{$\begin{array}{c}\text { Ran } \\
g o\end{array}$} & \multirow{2}{*}{$\begin{array}{c}\text { Mini } \\
\text { mo }\end{array}$} & \multirow{2}{*}{$\begin{array}{l}\text { Máx } \\
\text { mo }\end{array}$} & \multirow{2}{*}{ Media } & \multirow{2}{*}{$\begin{array}{l}\text { Desv. } \\
\text { Desviación }\end{array}$} & \multicolumn{2}{|c|}{ Asimetria } & \multirow{2}{*}{$\begin{array}{l}\text { Orden } \\
\text { según } \\
\text { la } \\
\text { Media } \\
\end{array}$} \\
\hline & & & & & & & & Estadistico & $\begin{array}{l}\text { Desv. } \\
\text { Error }\end{array}$ & \\
\hline \multirow{8}{*}{ 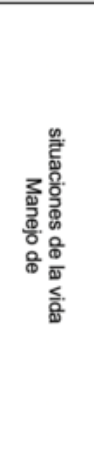 } & $\begin{array}{l}\text { 13.Aprender y } \\
\text { trabajar sola }\end{array}$ & 76 & 4 & 1 & 5 & 4,05 & 0,847 & $-1,184$ & 0,276 & 10 \\
\hline & $\begin{array}{l}\text { 14.Aprender y } \\
\text { trabajar en equipo }\end{array}$ & 76 & 3 & 2 & 5 & 4,18 & 0,86 & $-0,886$ & 0,276 & 7 \\
\hline & $\begin{array}{l}\text { 15.Afrontar } \\
\text { conflictos de } \\
\text { manera positiva }\end{array}$ & 76 & 4 & 1 & 5 & 3,51 & 1,064 & $-0,41$ & 0,276 & 22 \\
\hline & $\begin{array}{l}\text { 16. Negociación y } \\
\text { solución de } \\
\text { conflictos }\end{array}$ & 75 & 4 & 1 & 5 & 3,65 & 1,084 & $-0,571$ & 0,277 & 21 \\
\hline & $\begin{array}{l}\text { 17.Propononerse } \\
\text { objetivos respecto a } \\
\text { un proyecto }\end{array}$ & 75 & 2 & 3 & 5 & 4,2 & 0,805 & $-0,383$ & 0,277 & 6 \\
\hline & $\begin{array}{l}\text { 18.Visión de } \\
\text { solución de } \\
\text { problemas }\end{array}$ & 76 & 3 & 2 & 5 & 4 & 0,849 & $-0,403$ & 0,276 & 11 \\
\hline & $\begin{array}{l}\text { 19. Elaborar y llevar } \\
\text { a la práctica nuevas } \\
\text { ideas }\end{array}$ & 76 & 4 & 1 & 5 & 4,21 & 0,884 & $-1,144$ & 0,276 & 5 \\
\hline & $\begin{array}{l}\text { 20.Administrar el } \\
\text { teempo de manera } \\
\text { efectiva }\end{array}$ & 76 & 4 & 1 & 5 & 3,78 & 0,974 & $-0,775$ & 0,276 & 20 \\
\hline \multirow{6}{*}{ 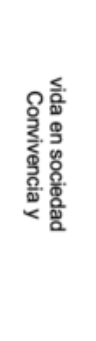 } & & 76 & 3 & 2 & 5 & 3,79 & 0,805 & $-0,225$ & 0,276 & 19 \\
\hline & $\begin{array}{l}\text { 21.Decidir y actuar } \\
\text { con juicio critico } \\
\text { frente a valores } \\
\text { an }\end{array}$ & & & & & & & & & \\
\hline & $\begin{array}{l}\text { implicaciones } \\
\text { sociales }\end{array}$ & 76 & 3 & 2 & 5 & 4,22 & 0,793 & $-0,755$ & 0,276 & 4 \\
\hline & $\begin{array}{l}\text { 23.Participar en el } \\
\text { desarrollo de la } \\
\text { localidad }\end{array}$ & 76 & 4 & 1 & 5 & 3,93 & 0,854 & $-0,796$ & 0,276 & 15 \\
\hline & $\begin{array}{l}\text { 24.Actuar con } \\
\text { respecto a la } \\
\text { diversidad } \\
\text { sociocultural } \\
\text { 25.Reconocer }\end{array}$ & 74 & 3 & 2 & 5 & 3,84 & 0,777 & $-0,248$ & 0,279 & 17 \\
\hline & $\begin{array}{l}\text { tradiciones de su } \\
\text { comunidad }\end{array}$ & 76 & 3 & 2 & 5 & 3,84 & 0,865 & $-0,066$ & 0,276 & 18 \\
\hline
\end{tabular}

Tabla 2

Resultado de escala Likert Competencias Integrales - Manejo de Situaciones y Convivencia Fuente: elaboración propia.

Los anteriores resultados dan una visión general del autoreporte realizado por las encuestadas, mostrando valores promedio para los 22 reactivos que indagan por las competencias integrales, que van desde 3,51 hasta 4,3, dentro de un máximo de 5 . La desviación estándar promedio también para los 22 reactivos fue 0,88 y el coeficiente de asimetría global fue $-0,785$.

Para el análisis de los resultados, los autores del instrumento proponen cuatro escalas de valoración que contienen los puntajes obtenibles y su correspondiente interpretación, como pueden verse en la tabla 3 . La sumatoria de promedios obtenidos para cada conjunto de competencias que componen cada competencia da un valor global de la misma, 
Tabla 3

Escalas de valoración de los resultados obtenidos con el cuestionario de competencias integrales de Suárez y otros (2012).

Fuente: elaboración propia con base en lo indicado por los autores del instrumento.

\begin{tabular}{|c|c|c|c|c|}
\hline \multicolumn{5}{|c|}{ Competencias } \\
\hline $\begin{array}{c}\text { Aprendizaje } \\
\text { permanente. } \\
\text { Puntajes }\end{array}$ & $\begin{array}{c}\text { Manejo de } \\
\text { información. } \\
\text { Puntajes }\end{array}$ & $\begin{array}{c}\text { Manejo de } \\
\text { situaciones de la } \\
\text { vida. } \\
\text { Puntajes }\end{array}$ & $\begin{array}{c}\text { Convivencia y } \\
\text { vida en } \\
\text { sociedad. } \\
\text { Puntajes } \\
\end{array}$ & Interpretación \\
\hline $20-25$ & $17-20$ & $33-40$ & $21-25$ & Muy alto \\
\hline $16-20$ & $13-16$ & $25-32$ & $16-20$ & Alto \\
\hline $11-15$ & $9-12$ & $17-24$ & $11-15$ & Medio \\
\hline $6-10$ & $5-8$ & $9-16$ & $6-10$ & Bajo \\
\hline $0-5$ & $0-4$ & $0-8$ & $0-5$ & Muy bajo \\
\hline
\end{tabular}

Tabla 3

Escalas de valoración de los resultados obtenidos con el cuestionario de competencias integrales de Suárez y otros (2012).

Fuente: elaboración propia con base en lo indicado por los autores del instrumento.

Con base en datos recabados se presentan, en la Tabla 4, los hallazgos por competencias para los cuatro grupos por los que indaga el instrumento.

Tabla 4

Competencias integrales y tecnológicas- escala Likert.

Fuente: elaboración propia.

\begin{tabular}{lcc}
\hline \multicolumn{1}{c}{ Competencia } & $\begin{array}{c}\text { Sumatoria de } \\
\text { los promedios }\end{array}$ & $\begin{array}{c}\text { Puntaje máximo posible } \\
\text { según número de reactivos }\end{array}$ \\
\hline Manejo de Situaciones de la Vida (MS) & 31 & 40 \\
$\begin{array}{l}\text { Para la convivencia y la vida en sociedad } \\
\text { (CVS) }\end{array}$ & 20 & 25 \\
Logro del Aprendizaje Permanente (AP) & 20 & 25 \\
Manejo de la Información (MI) & 17 & 20 \\
\hline
\end{tabular}

Tabla 4

Competencias integrales y tecnológicas- escala Likert.

Fuente: elaboración propia.

A partir de las escalas ya mencionadas, el resultado global en las cuatro competencias está entre muy alto y alto. Muy alto, con 17 puntos que representan el $85 \%$ del máximo posible, en manejo de información. Alto, con 20 puntos que representan el $80 \%$, en dos competencias: logro del aprendizaje permanente y convivencia, y vida en sociedad. Y alto, con 31 puntos que representan el 77\%, para el manejo de situaciones de la vida.

El promedio más alto reportado corresponde al de competencias de analizar, sintetizar, utilizar y compartir información, con un valor de 4,3 y el más bajo, 3,51, corresponde a competencias para afrontar conflictos de manera positiva. En la tabla 5 se pueden observar los puntajes máximos y mínimos obtenidos. Todos los promedios oscilan entre 3,51 y 4,3. 
Tabla 5

Máximos y mínimos por afirmación - Cuestionario de competencias integrales y tecnológicasescala Likert. Fuente: elaboración propia.

\begin{tabular}{lc}
\multicolumn{1}{c}{ Competencia } & Promedio Obtenido \\
\hline Máximos: & 4,3 \\
Analizar, sintetizar, utilizar y compartir información & 4,28 \\
Búsqueda e identificación de información & 4,24 \\
$\begin{array}{l}\text { Aprender, asumir y dirigir el propio aprendizaje a lo largo de la } \\
\text { vida }\end{array}$ & 4,22 \\
$\begin{array}{l}\text { Tomar en cuenta las implicaciones sociales del uso de la } \\
\text { tecnología }\end{array}$ & 4,21 \\
$\begin{array}{l}\text { Para elaborar nuevas ideas y llevarlas a la práctica } \\
\text { Proponerse objetivos con el fin de conseguir lo previsto }\end{array}$ & 4,20 \\
mediante la planificación de un proyecto & \\
Mínimos: & 3,51 \\
Afrontar conflictos de manera positiva & 3,65 \\
Negociación y solución de conflictos & 3,78 \\
Administrar el tiempo de manera efectiva & 3,79 \\
$\begin{array}{l}\text { Decidir y actuar con juicio crítico con respecto a los valores y } \\
\text { normas sociales y culturales. }\end{array}$ &
\end{tabular}

\section{Tabla 5}

Máximos y mínimos por afirmación - Cuestionario de competencias integrales y tecnológicas- escala Likert.

Fuente: elaboración propia.

Vistos estos mismos resultados desde otro criterio de presentación, como la distribución de frecuencias de las estudiantes, considerando los rangos establecidos por Suárez y otros (2012) para cada competencia, se obtuvo que la mayoría de las estudiantes se ubicó en la escala alto y muy alto, como se observa en la figura 1 y en la tabla 6 .

\section{Resultado del cuestionario de compentencias integrales y tecnológicas por} competencia y por niveles

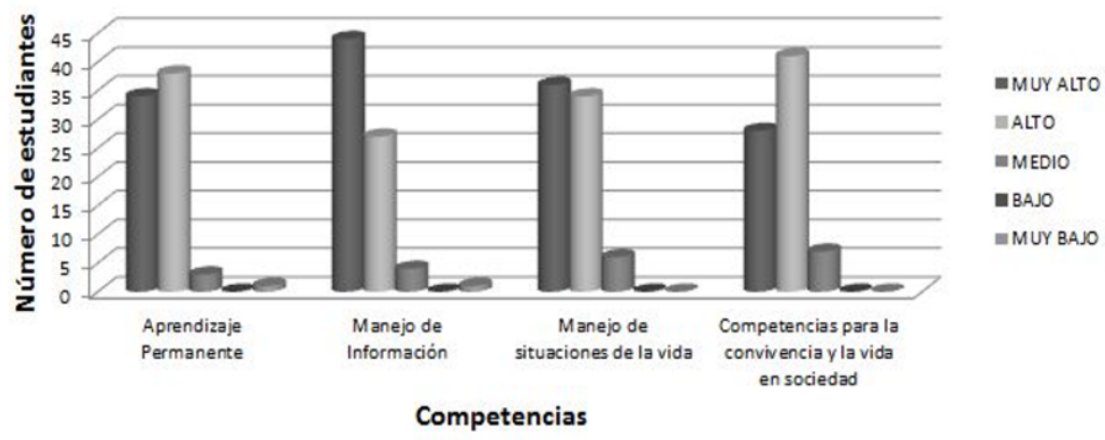

Figura 1.

Distribución de frecuencias de resultados obtenidos según escala Likert.

Fuente: elaboración propia. 
Tabla 6

Distribución de frecuencias de los resultados obtenidos según escala Likert

Fuente: elaboración propia.

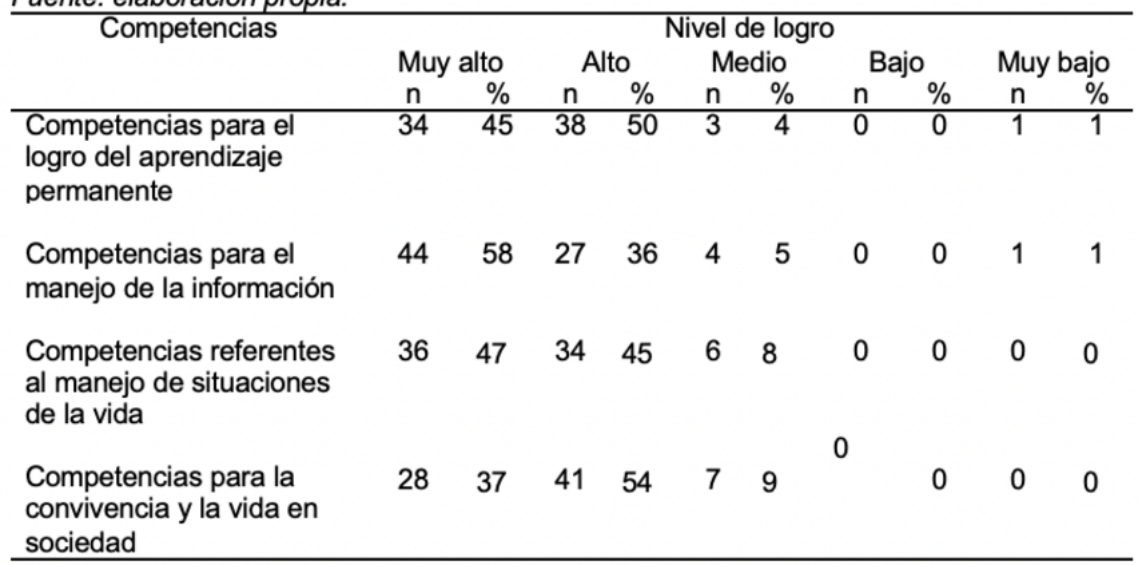

\section{Tabla 6}

Distribución de frecuencias de los resultados obtenidos según escala Likert

Fuente: elaboración propia.

Los resultados permiten ver que más del $90 \%$ de las estudiantes se ubica entre los dos primeros niveles de logro de la competencia. Lo anterior significa que las estudiantes están recibiendo en las asignaturas en las cuales incorporan TIC no solo conocimiento disciplinar, sino que están desarrollando su capacidad de aprender de manera autorregulada, para asumir y dirigir el propio aprendizaje a lo largo de la vida; también se está potenciando su formación en autonomía y apertura hacía el conocimiento. En cuanto a las competencias para el manejo de información, se ven a sí mismas con buenas habilidades y destrezas para la búsqueda, manejo y comunicación de información. En cuanto estas últimas, se destaca que, por medio de trabajos colaborativos realizados por pares, las estudiantes buscan y seleccionan recursos que luego comparten mediante presentaciones en la plataforma Edmodo.

En cuanto a la confiabilidad de esta primera parte del estudio, se calculó con el programa SPSS el alfa de Cronbach con el siguiente resultado global: 0,895 , considerando los 22 elementos o reactivos de la escala Likert. Para este mismo índice, por cada uno de los grupos de variables se obtuvo el resultado que se observa en la tabla 7 : 
Tabla 7

Coeficiente alfa de Cronbach obtenido para las variables de la escala Likert.

Fuente: elaboración propia.

\begin{tabular}{|c|c|c|}
\hline Competencias & $\begin{array}{l}\text { Número de } \\
\text { Ítems }\end{array}$ & Alfa de Cronbach \\
\hline Aprendizaje permanente & 5 & 0,82 \\
\hline Manejo de información & 4 & 0,78 \\
\hline Manejo de situaciones de la vida & 8 & 0,713 \\
\hline Convivencia y vida en sociedad & 5 & 0,693 \\
\hline
\end{tabular}

Tabla 7

Coeficiente alfa de Cronbach obtenido para las variables de la escala Likert Fuente: elaboración propia.

\section{Resultados del segundo objetivo correspondiente al componente cualitativo}

El objetivo fue: Identificar beneficios que reportan las estudiantes de séptimo grado de $\mathrm{BS}$, como obtenidos mediante el uso de los REA en actividades de aprendizaje colaborativo utilizando TIC, en desarrollo de habilidades comunicativas orales, interacción con el otro, valoración de las tutorías, ayuda mutua.

A la muestra de 76 estudiantes, ya mencionada, se aplicó el instrumento de Martínez y otros (2013), con adaptaciones menores. Algunos resultados se aprecian en las figuras 2 y 3 .

Figura 2. Respuesta a la pregunta sobre relación del aprendizaje en equipo y el entorno laboral futuro.

Entre los resultados obtenidos están: el 68\% considera que el aprendizaje colaborativo es adecuado para desarrollar las destrezas orales y escritas por igual. También el $85 \%$ da mucha importancia a las tutorías. Como principales aspectos positivos de aprendizaje en grupo se obtuvo: $53 \%$ un mejor aprendizaje, el $22 \%$ resaltó la ayuda mutua y el $25 \%$ el compañerismo y la oportunidad de relacionarse. En cuanto a aspectos negativos: el $30 \%$ no encontró, el 25\% reportó desorden, el 23\% discusiones y desacuerdos, el $11 \%$ estudiantes que no trabajan. El 59\% considera que el aprendizaje colaborativo reviste igual importancia en primaria o en secundaria.

Las estudiantes reconocen la importancia del aprendizaje colaborativo en el momento actual y en el futuro, expresan que permite compartir saberes con sus pares, lograr mayor efectividad en el aprendizaje y desarrollar sus habilidades comunicativas escritas y orales. Lo anterior encuentra sustento en lo planteado por García-Varcárcel, Hernández y Muñoz-Repiso (2012), quien plantea el reconocimiento de las teorías de aprendizaje actuales sobre la interacción con el otro en el aprendizaje. En general hay buena aceptación y conciencia de diversas ventajas del aprendizaje colaborativo.

Sin embargo, de la información recabada sobre aspectos negativos del aprendizaje colaborativo, se concluye que se debe trabajar para potenciar 
las habilidades de resolución de conflictos y motivar hacia la participación responsable y equitativa.

Las estudiantes entrevistadas encuentran en el aprendizaje colaborativo una oportunidad para interactuar con sus compañeras, mejorar sus conocimientos, realizar aportes, hacer consultas al docente y buscar solución a las dificultades que se les presentan, dentro de un ambiente cordial y respetuoso. Son conscientes de la importancia de la búsqueda del bien común y de su contribución para lograrlo. Sin embargo, algunas pocas expresan que no todas colaboran pudiendo hacerlo.

Siguiendo a García-Varcárcel y otros (2013), quienes plantean que el aprendizaje colaborativo se fundamenta en la teoría constructivista, otorgándole un papel protagónico al alumno, unido con frases recurrentes de las respuestas en las entrevistas, llevó a establecer las categorías de interacción y bien común con indicadores de contacto y retroalimentación, identificando beneficios en el proceso de aprendizaje. Se encontró también que al escucharse y ser escuchadas, las estudiantes se motivan por ser tenidas en cuenta.

\section{Resultados del tercer objetivo correspondiente al componente cualitativo}

El objetivo fue: Inferir características de REA en el aprendizaje de matemáticas orientados al desarrollo de CI en recuperación de información, comprensión y aprendizaje de conceptos, empleo de algoritmos y motivación por el aprovechamiento pedagógico de la tecnología, que reconocen las estudiantes de séptimo grado de EBS, como vivenciadas con su uso interactivo.

Las estudiantes entrevistadas han encontrado en los REA elementos motivadores, pues mientras interactúan con el recurso van aprendiendo o, porque es lúdico, les presenta en otra forma los contenidos, de manera atractiva visualmente y pueden realizar ejercicios. Les permite tener el control, como en el caso de los videos, para devolverlos en los apartes que no comprendan bien. Les permite explorar distintos niveles de dificultad. Lo anterior da cuenta de la utilidad, la motivación y la autorregulación.

Sin embargo, para algunas pocas estudiantes se vio la necesidad de acompañar más de cerca el proceso mediante refuerzos, pues se encontró que en algunos casos no consiguen la meta propuesta para la actividad o la estudiante realiza los ejercicios, pero no tiene una buena comprensión de lo realizado.

\section{DISCUSIÓN Y RECOMENDACIONES}

Los hallazgos en cuanto a motivación, autorregulación y utilidad encuentran sustento en autores como Mortera (2011) y Pazos y otros (2015). Al respecto de la tranquilidad que les produce tener el control del video y poder volver a ver aquello que no han entendido, Perrenud (2009) plantea que las instituciones educativas se han limitado a proporcionar saberes a solo un porcentaje reducido de estudiantes. Desde este punto 
de vista, los REA incorporados adecuadamente al currículum son una alternativa para disminuir la brecha mencionada. La utilidad de los REA es reconocida por las estudiantes y ha sido objeto de muchos estudios y documentada en escritos como el de Ramírez (2012).

De otra parte, las estudiantes reportaron en las entrevistas que con el uso de REA pueden comprender diversos temas y pueden recuperar información acerca de conceptos o bases de los mismos. Pueden ajustarse a su propio ritmo de aprendizaje y abrir la mente para considerar distintas alternativas de solución. Los REA utilizados permitieron realizar ejercicios interactivos, algunos con respuesta e información complementaria, de modo que sirviera de elemento de retroalimentación y reflexión desde el aprendizaje centrado en al alumno.

Con referencia a la educación apoyada en recursos tecnológicos, Avilés y otros (2010), se refieren a dichas tecnologías como escenarios cognitivos verdaderos, que permiten involucrar de manera significativa al estudiante, siendo él el centro de su propio aprendizaje. Subrayan que la reflexión y la indagación son componentes centrales de este nuevo proceso. De las entrevistas se desprende que las estudiantes manifestaron interés por el aprendizaje y agrado porque, aunque en un primer momento no hayan entendido, con la práctica y el estudio autorregulado lograron avances.

Rodríguez y Saldaña (2010) plantean que los estudiantes deben aplicar el razonamiento lógico matemático ante situaciones de la vida diaria, pero que para ello el aprendizaje debe iniciarse en la vida escolar. Lograr motivar e involucrar a las estudiantes en la resolución de problemas matemáticos de manera activa es un paso hacia esta meta. Sin embargo, para conseguir mejores resultados y conjugar distintos aspectos que conducen a la formación integral como el aprendizaje colaborativo se requiere formación docente apropiada.

Desde el planteamiento del MEN (2006), estándares de competencias matemáticas, grados sexto y séptimo, la investigación permitió identificar el ejercicio de las siguientes competencias:

o Predigo y comparo los resultados de aplicar transformaciones rígidas, (traslaciones, rotaciones, reflexiones) sobre figuras bidimensionales en situaciones matemáticas (GeoGebra).

o Conjeturo acerca de los resultados de un experimento aleatorio utilizando nociones básicas de probabilidad.

o Resuelvo problemas en contextos de medidas relativas y de variaciones de medidas.

De esta manera se concluye que los tres objetivos específicos se cumplieron, permitiendo identificar en este estudio competencias directamente relacionadas con ambientes de aprendizaje mediados por tecnologías, aprendizaje colaborativo y competencias matemáticas.

De acuerdo con los resultados anteriores, la validación de los mismos mediante triangulación y el análisis realizado, se puede decir que se evidenció que la mayoría de las estudiantes desarrolla la competencia de apropiación de tecnología, específicamente: "utilizo las tecnologías de la información y la comunicación para apoyar mis procesos de aprendizaje 
y actividades personales (recolectar, seleccionar, organizar y procesar información)" (MEN, 2008, p. 20).

Comparación de resultados obtenidos para algunas subcompetencias de la escala Likert y la información recabada a partir de las entrevistas:

Se observó que los resultados son congruentes y que ambos conducen al reconocimiento de la utilidad de las TIC, en particular de los REA, la motivación que genera, la utilidad que representan y la posibilidad de utilizarlos dentro del aprendizaje autorregulado. Promedios de 4,24/5 y $4,2 / 5$ en subcompetencias relativas a asumir y dirigir el propio aprendizaje a lo largo de la vida, por ejemplo, permiten pensar en esta congruencia.

Las estudiantes entrevistadas encuentran en el aprendizaje colaborativo una oportunidad para interactuar con sus compañeras, mejorar sus conocimientos, realizar aportes, hacer consultas al docente y buscar solución a las dificultades que se les presentan, en la mayoría de los casos dentro de un ambiente cordial y respetuoso. Son conscientes de la importancia de la búsqueda del bien común y de su contribución para lograrlo. Sin embargo, algunas pocas expresan que no todas colaboran pudiendo hacerlo. En la subcompetencia trabajo en equipo de la escala Likert se obtuvo un promedio de 4,18 /5.

En cuanto a bondades y ventajas del aprendizaje colaborativo en sentido positivo, revelan que se favorece la interacción y la comunicación, como lo plantea García-Varcárcel (2012), y que los REA agregan un componente motivacional al aprendizaje colaborativo. La encuesta mostró en cuanto a aspectos negativos, reportado por el 23\%: desorden, disgustos y estudiantes que no trabajan. Estos son aspectos sobre los cuales el docente debe buscar estrategias para involucrar al estudiante.

De esta manera se concluye que los tres objetivos específicos se cumplieron, permitiendo identificar en este estudio competencias directamente relacionadas con ambientes de aprendizaje mediados por tecnologías, aprendizaje colaborativo y competencias matemáticas. Estas competencias fueron expresadas en términos de categorías e indicadores, pero también están enmarcadas dentro de los estándares oficiales del MEN (2006).

\section{Validez}

La información recabada en el trabajo de campo para la investigación cualitativa ha sido validada mediante triangulación, contrastando resultados obtenidos para cada categoría, comparando lo obtenido de las entrevistas con el resultado de la observación y el contraste con la literatura. La observación fue contrastada con los docentes de las clases observadas. En lo cuantitativo, sobre la validez interna, se cuidaron los aspectos que plantean Valenzuela y Flórez (2012), como maduración, sensibilización a los test e instrumentación deficiente. Por ser investigación transeccional descriptiva, otros riesgos que relacionados con la manipulación de variables no aplican. 
Todos los instrumentos utilizados están validados por estudios previos. Se atendió a los principios éticos y se obtuvieron los consentimientos informados para la participación voluntaria.

La pregunta inicial fue: ¿Cuál es el efecto del uso de REA en matemáticas mediados por TIC que reportan las estudiantes del IGM sobre su formación integral en séptimo grado de educación básica secundaria $(\mathrm{EBS})$ ?

Los resultados de la aplicación de instrumentos y su análisis, incluyendo la triangulación, mostraron que las estudiantes reconocen que el uso pedagógico de los REA en matemáticas en grado séptimo permite el fortalecimiento de competencias integrales, pues incrementa la motivación, facilita el aprendizaje, promueve la autorregulación, fomenta el desarrollo del razonamiento lógico matemático, la interacción entre los educandos y con el docente, el sentido de pertenencia a una comunidad. Estos aprendizajes y actitudes se ubican dentro de los estándares de competencias del MEN (2006), como se ha indicado anteriormente en este capítulo.

Como sugerencia para futuros estudios, se plantea profundizar y buscar estrategias para fortalecer las competencias de resolución de conflictos y negociación, ya que a nivel de detalle de la escala Likert, las dos competencias mencionadas fueron las que reportaron un resultado más bajo.

\section{REFERENCIAS}

1. Alvarado, S. (2012). Las Tic como apoyo de mediación pedagógica en la formación de competencias ciudadanas en niños del tercer grado de preescolar (Tesis de pregrado). Universidad Autónoma de Bucaramanga, Bucaramanga, Colombia e Instituto Tecnológico de Monterrey, Monterrey, México.

2. Aragón, E., Castro, C., Gómez, B. y González, R. (2009). Objetos de aprendizaje como recursos didácticos para la enseñanza de las Matemáticas. Apertura, 1(1), 1-13. Recuperado de: http://www.redalyc.o $\mathrm{rg} /$ articulo.oa?id $=68820815008$

3. Asprilla, F. (2012). Relación entre el uso de REAS y la competencia interpretativa de estudiantes de Básica Secundaria en lengua castellana (Tesis de pregrado). Universidad Autónoma de Bucaramanga, Bucaramanga, Colombia e Instituto Tecnológico de Monterrey, Monterrey, México.

4. Avilés, M., Díaz, J., Esquivel, S. y Hernández, G. (2010). Apoyo en el aprendizaje: REA, una opción tecnológica para el desarrollo de competencias en Geometría y Trigonometría a nivel bachillerato. En J. V. Burgos y M. S. Ramírez (coord.), Recursos Educativos Abiertos en Ambientes Enriquecidos con Tecnología (361-381). Monterrey, México.: Tecnológico de Monterrey. Recuperado de: http://catedra.ruv.itesm.mx/ bitstream/987654321/566/8/ebook

5. Daza-Orozco, CE. (2015). La Investigación como proyecto de Vida: Un acercamiento al quehacer de los Semilleros de Investigación. Corporación 
Internacional para el Desarrollo Educativo - CIDE. https://doi.org/10.1 3140/RG.2.1.2631.1761

6. Delors, J. (Ed.) (1996). Informe a la UNESCO de la Comisión Internacional sobre la Educación para el siglo XXI. En la educación encierra un tesoro. Los cuatro pilares de la educación (90-103). México.

7. Díaz-Barriga, A. (2011). Competencias en educación. Corrientes de pensamiento e implicaciones para el currículo y el trabajo en el aula. Revista Iberoamericana de Educación Superior, 2(5), 3-24. Recuperado de:

9. Dzul, M. (2013). Aplicación básica de los métodos científicos. Diseño no experimental. Hidalgo, México: Universidad Autónoma del Estado de Hidalgo. Recuperado de: http://www.uaeh.edu.mx/virtual

10. Fernández-Cárdenas, J. (2011, Junio 23). Multimodalidad y calidad educativa: Los retos de la construcción de conocimiento disciplinar en ambientes mediados por tecnología digital. Ponencia presentada en el XII Encuentro Internacional Virtual Educa, México, D. F.

11. García-Valcárcel, A., Hernández, M., Muñoz-Repiso, A. (2012). La metodología del aprendizaje colaborativo a través de las TIC: una aproximación a las opiniones de profesores y alumnos. Revista Complutense de Educación, 23(1), 161-188. Recuperado de: http://revist as.ucm.es/index.php/RCED/article/view/39108

12. Gardner, H. (1988). La nueva ciencia de la mente. Historia de la revolución cognitiva. Barcelona, España: Ingraf, S.A.

13. Gates, B. (1995). Camino al futuro. Bogotá, Colombia: McGraw-Hill interamericana, S. A.

14. Hernández, Fernández y Baptista (2010). Metodología de la investigación. (5`. Ed.). Perú: Empresa Editora El Comercio S.A.

15. Jaramillo, P. y Ruíz, M. (2010). El desarrollo de la autonomía: más allá del uso de las TIC para el trabajo independiente. Revista Colombiana de Educación, (58), 78-95. Recuperado de: http://dialnet.unirioja.es/servlet /articulo?codigo $=4155474$

16. Ley General de Educación. (1994). Ley 115 de 1994. Bogotá, Colombia: Imprenta Nacional.

17. López, J. (2014).Enseñary aprender competencias. Málaga, España: Ediciones Aljibe.

18. López, O. (2010). Presentación. Revista Colombiana de Educación, (58), 9-11. Recuperado de: http://revistas.pedagogica.edu.co/index.php/RCE /article/view/646/638

19. López, O. y Hederich, C. (2010). Efecto de un andamiaje para facilitar el aprendizaje autorregulado en ambientes hipermedia. Revista Colombiana de Educación, (58), 14-39. Recuperado de: http://www.academia.edu/4785744/Efecto_de_un_andamiaje_para _facilitar_el_aprendizaje_autorregulado_en_ambientes_hipermedia

20. Martínez, M., Catalá, P.y Díaz, M. (2013).Aprendercolaborando: Estrategias de aprendizaje colaborativo integradas en el aula universitaria. Recuperado de: https://web.ua.es/es/ice/jornadas-redes/documentos/2013-comunic aciones-orales/328708.pdf

21. MEN. Ministerio de Educación Nacional. (2005). Un mundo de competencias. ¿Qué son? Colombia Aprende, Bogotá, Colombia. 
Recuperado de: http://www.colombiaaprende.edu.co/html/competenci as/1746/w3-article-249280.html.

22. MEN. Ministerio de Educación Nacional. (2010). Revolución educativa. Programa para el desarrollo de competencias. Bogotá. Colombia. Recuperado de: https://www.mineducacion.gov.co/1759/articles-21759 6_archivo_pdf_desarrollocompetencias.pdf

23. MEN. Ministerio de Educación Nacional. (2006). Estándares Básicos de Competencias en Matemáticas, Bogotá, Colombia. Recuperado de: http:// www.mineducacion.gov.co/1621/articles-340021_recurso_1.pdf

24. MEN. Ministerio de Educación Nacional. (2008). Ser competente en tecnologia: ¡Una necesidad para el desarrollo! Guia No. 30, Bogotá, Colombia: Imprenta Nacional de Colombia.

25. MEN. Ministerio de Educación Nacional. (2010). Acciones y lecciones. Revolución educativa 2002-2010. Bogotá, Colombia.

26. Morin, E. (1999). Los siete saberes necesarios para la educación del futuro. París: UNESCO.

27. Mortera, F. (2011). Uso de recursos educativos abiertos para mejorar las prácticas docentes y habilidades digitales: Metodología de Búsqueda y adopción de Recursos Educativos Abiertos. Revista de Investigación Educativa Escuela de Graduados en Educación, 2(4), 19-28. Recuperado de: http://rieege.tecvirtual.mx/index.php/rieege/article/view/4

28. Naeemi, F., Zare, H., Hormozi, M., Shaqaqi, F. y Kaveh, M. (2011). Designing and Implementing a Situated Learning Program and Determining its Impact on the Students' Motivation and Academic Achievement. Studies in Learning \& Instruction, 3(1), 27-30. Recuperado de: http://www.sid.ir/en/VEWSSID/J_pdf/126520116007.pdf

29. OCDE. (2005). La Definición y Selección de Competencias Clave Resumen. Deseco. Recuperado de: http://deseco.ch/bfs/deseco/en/index /03/02.html

30. OCDE. (2013). PISA 2012. Results in Focus. Recuperado de: http://www. oecd.org/pisa/keyfindings/pisa-2012-results-overview.pdf

31. OCDE. (2016). PISA 2015. Resultados Clave. Recuperado de: https://ww w.oecd.org/pisa/pisa-2015-results-in-focus-ESP.pdf

32. Pazos, L., Tenorio, G. y Ramírez, M. (2015). Atributos de la innovación en el marco del movimiento educativo abierto para desarrollar competencias matemáticas. Revista Actualidades Investigativas en Educación, 15(3), 1-24. Recuperado de: https://repositorio.itesm.mx/ortec/bitstream/112 85/610642/1/2015-Pazos-Tenorio-Ramirez.pdf

33. Pereira, Z. (2011). Los diseños de método mixto en la investigación en educación: Una experiencia concreta. Revista electrónica Educare, 15(1), 15-29. Recuperado de: www.redalic.org/html/1941/194118804003

34. Ramírez, H., Huerta, A. Flores, M. (2012). Impacto del nuevo modelo educativo del Instituto Politécnico Nacional en el desarrollo de la práctica docente de los profesores de la Escuela Superior de Ingeniería Mecánica y Eléctrica. Revista de Investigación Educativa de la Escuela de Graduados, (5), 27-35. Recuperado de: http://rieege.tecvirtual.mx/index.php/rieege/ article/download/39/34

35. Ramírez, M. (2012). Modelos y estrategias de enseñanza para ambientes innovadores. Monterrey, México: Editorial Digital Tecnológico de Monterrey. 
36. Rodríguez, C. y Saldaña. B. (2010). Estrategias de enseñanza que favorecen el razonamiento lógico matemático en alumnos de primaria, mediante la implementación de REA. En J. V. Burgos y M. S. Ramírez (coord.), Recursos Educativos Abiertos en Ambientes Enriquecidos con Tecnología (85-97). Monterrey, México.: Tecnológico de Monterrey. Recuperado de http://catedra.ruv.itesm.mx/bitstream/987654321/566/8/ebook

37. Suárez, D., Rodríguez, E. y Rodríguez, C. (2012). Cuestionario de Competencias Integrales y Tecnológicas. (Nivel de Secundaria, Media Superior, Universitario y Postgrado)

38. Tobón, S. (2004). Formación basada en competencias. Pensamiento complejo, diseño curricular y didáctica. Bogotá, Colombia: Esfera Editores Ltda.

39. Valenzuela, J. y Flórez M. (2012). Fundamentos de Investigación Educativa Vol 2 y 3. Monterrey, México: Editorial Digital Tecnológico de Monterrey. 